\title{
Incidence and predictors of lost to follow-up among drug-resistant tuberculosis patients at University of Gondar Comprehensive Specialized Hospital, Northwest Ethiopia: a retrospective follow-up study
}

Getahun Molla Kassa* (10, Alemayehu Shimeka Teferra, Haileab Fekadu Wolde, Atalay Goshu Muluneh and Mehari Woldemariam Merid

\begin{abstract}
Background: The emergence of Drug-Resistance Tuberculosis (DR-TB) is an increasing global public health problem. Lost to Follow-up (LTFU) from DR-TB treatment remains a major barrier to tuberculosis epidemic control and better treatment outcome. In Ethiopia, evidences on the incidence and predictors of LTFU are scarce. Thus, this study aimed to determine the incidence and identify the predictors of LTFU among DR-TB patients.

Methods: A retrospective follow-up study was conducted among a total of 332 DR-TB patients at the University of Gondar comprehensive specialized hospital. Data were retrieved from patient records from September 2010 to December 2017 and entered in to Epi-data 4.2.0.0 and analysed using Stata14.1 software. The risk was estimated using the Nelson-Aalen cumulative hazard curve. A log-rank test was used for survival comparisons between categories of independent variables. The Gompertz regression model was fitted, and hazard ratio with a 95\% confidence interval $(\mathrm{Cl})$ was used to measure the strength of associations. Variables with less than $0.05 p$-values in the multivariable model were considered as significantly associated with LTFU.

Results: Among a total of 332 patient records reviewed, 206 (62.05\%) were male. The median age was 30 years (Inter Quartile Range (IQR): 23-40). Forty-one (12.35\%) of the participants had no history of TB treatment, while a quarter of were TB-HIV co-infected. Closely all (92.17\%) of the patients had pulmonary tuberculosis. The median follow up time was 20.37 months (IQR: 11.02, 21.80). Thirty-six (10.84\%) patients were lost from follow-up with an incidence rate of 6.47 (95\% Cl: 4.67, 8.97)/1000 Person Months (PM). Homelessness (Adjusted Hazard Ratio (AHR) = 2.51,95\%Cl: 1.15, 5.45) and treatment enrolment year from 2013 to $2014(\mathrm{AHR}=3.25,95 \% \mathrm{Cl}: 1.30,8.13$ ) were significant predictors of LTFU.

Conclusion: This study indicated that LTFU among DR-TB registered patients was high in the first six months compared to subsequent months. Homelessness and year of treatment enrolment were independent predictors of $L T F U$, requiring more economic support to patients in order to ensure treatment completion. This result can be generalized to patients who are using DR-TB treatment in similar settings.
\end{abstract}

Keywords: Drug-resistant tuberculosis, Incidence, Predictors, Lost to follow-up, Retrospective study

\footnotetext{
* Correspondence: getahunm8@gmail.com

Department of Epidemiology and Biostatistics, Institute of Public Health,

College of Medicine and Health Sciences and Specialized Comprehensive

Hospital, University of Gondar, Gondar, Ethiopia
}

(c) The Author(s). 2019 Open Access This article is distributed under the terms of the Creative Commons Attribution 4.0 International License (http://creativecommons.org/licenses/by/4.0/), which permits unrestricted use, distribution, and reproduction in any medium, provided you give appropriate credit to the original author(s) and the source, provide a link to the Creative Commons license, and indicate if changes were made. The Creative Commons Public Domain Dedication waiver (http://creativecommons.org/publicdomain/zero/1.0/) applies to the data made available in this article, unless otherwise stated. 


\section{Background}

Tuberculosis (TB) is currently a significant public health problem worldwide [1]. The emergence of DR-TB, defined as resistance to at least one of the core first line anti-TB drugs, has become a threat to the global TB care and prevention [2]. According to the 2018 World Health Organization (WHO) TB report, an estimated 3.5\% of the new and $18 \%$ of the previously treated TB cases had Multi-Drug Resistant (MDR) or Rifampicin Resistant (RR) TB, respectively [3]. Nearly $50 \%$ of the global DRTB cases were found in China, India, and the Russian Federation [2-5]. Worldwide, 600,000 and 558,000 DRTB cases emerged in 2016 and 2017, respectively [3, 5].

In 2017, the estimated prevalence of MDR or RR TB in Africa was 2.7 and $14 \%$ among new and previously treated TB patients, respectively [3]. A meta-analysis in SubSaharan countries showed the pooled prevalence of DR$\mathrm{TB}$ among the new and the previously treated patients was estimated to be 12.6 and $27.2 \%$, respectively [6].

Ethiopia is one of the 30 high DR-TB burden countries globally and stands third among African countries with annual estimates of 2100 DR-TB cases from a total of annually notified TB cases [7]. Two DR-TB surveys in 2005 and 2014 and the 2018 WHO TB report in Ethiopia showed that the prevalence of DR-TB was 1.6, 2.3, and $2.7 \%$ among new TB cases, and 11.8, 17.8, and 14\% among previously treated ones, respectively $[3,7,8]$. According to one meta-analysis, the overall prevalence of MDR-TB among newly diagnosed and previously treated TB patients was 2 and 15\%, respectively [9]. DR-TB has been widely distributed throughout Ethiopia $[8,10]$.

Globally, TB incidence is falling by $2 \%$ annually; the fastest decline since 2010 has exceeded $4 \%$ per year in several high $\mathrm{TB}$ burden countries, including Ethiopia. However, to reach the first milestones of the "end TB strategy" by the year 2020, the annual reduction of TB incidence should be 4 to $5 \%$ [5].

Global DR-TB treatment success rate was nearly 50\% although some high burden countries including Ethiopia showed a treatment success rate of more than $75 \%$ and this low treatment success rate has been due to high LTFU and deaths $[2,3,11]$. Lost to follow-up from DRTB treatment remains a major barrier to the cure and epidemic control of DR-TB $[12,13]$.

According to a qualitative study done in India, developing short duration treatment regimens, reducing pill burden, and providing of motivational counseling [14] were the top identified themes that can reduce LTFU. Better general TB knowledge and higher levels of trust in clinical teams [15] were also protective factors against LTFU. Studies in Asia and Africa reported that adverse drug effects [14-16], ambulatory model of treatment initiation, and different providers in the intensive and continuation phase of treatment [17] were risk factors to LTFU.
The few studies done on treatment outcome and its determinants among patients with DR-TB in Ethiopia showed that poor treatment outcome was due to LTFU and death $[18,19]$. But there has been only limited evidence on the incidence and predictors of LTFU among DR-TB treatment patients in the country; despite the high burden of DR-TB cases. Therefore, estimating the rate and identifying the risk factors for LTFU is essential to prioritize the high risk groups of patients for clinical and programmatic interventions, to achieve the Sustainable Development Goals (SDGs) and the end TB strategy targets, to prevent the community from primary DR-TB infection, and to reduce further drug resistance developments. In addition, this study will also inform the respective stakeholders about the current state of LTFU among DR-TB patients, and assist in planning.

\section{Methods}

\section{Study design and setting}

An institution-based retrospective follow-up study was conducted on DR-TB patients taking treatments at the University of Gondar Comprehensive Specialized Hospital from September 2010 to December 2017.

The hospital is located in Gondar city, Amhara National Regional State, $738 \mathrm{~km}$ to the northwest of Addis Ababa, the capital of Ethiopia. The University of Gondar comprehensive specialized teaching hospital serves more than seven million people of North Gondar and neighbouring zones. It is the second oldest hospital that started DR-TB treatment in September 2010 s only to St. Peter's TB specialized hospital in the country and first in Amhara Region. Gondar has one treatment initiation and five treatment follow-up centres for DR-TB. There were also ten government and twelve public private mix DOTs clinics in the city. The hospital had two Xpert MTB/RIF assay machine, and external quality assured TB culture and acid fast staining sites for TB diagnosis. A total of 341 DR-TB patients were treated at the University of Gondar comprehensive specialized hospital from the beginning of the DR-TB treatment up to December 2017.

\section{Study population and data collection procedures}

The source population was all patients enrolled at the hospital for DR-TB treatment, while the study population included all 341 new drug-resistant tuberculosis patients registered form September 2010 to December 2017.

The data were collected from patient follow-up charts, registration books, and computer data base using a data extraction checklist which was prepared in English. The records to be reviewed were identified using patient identification numbers. The data were collected by three bachelor degree graduate nurses working at the TB ward under a close supervision of the principal investigator. In 
order to control the quality of data, training was given to data collectors on the objectives of the study and on how to extract data from patient records. The supervisor checked the completeness and consistency of the extracted data daily.

\section{Variables}

The outcome variable of this study was time in months from the start of treatment to LTFU or censored. Lost to follow-up was defined as a patient who took anti-TB treatment for any duration and interrupted it for two or more consecutive months for any reason; censored was defined when LTFU was not observed during the follow-up time which might include cured, treatment completed, treatment failed, treatment stopped, dead, transfer out and those who were still on treatment at the end of the study $[7,20]$ (Table 1).

Different characteristics at baseline were assessed from the registration document of the patients. The first characteristic assessed was socio-demographic which included age, sex, employment status, educational status, marital status, residence, region, and housing conditions. With regard to housing conditions, homeless was defined as patients who lived in streets or lacked fixed, regular, and adequate night-time residence. The second characteristics were behavioural components. These include smoking and alcohol drinking status. The third characteristics were clinical components, which included HIV co-infection, functional status, site of TB disease, baseline sputum smear microscopy result, culture conversion, presence of chronic complications, lung complication, DR-TB type, baseline $\mathrm{BMI}$ and $\mathrm{TB}$ treatment history. TB treatment history was classified as previously treated and untreated cases. Previously treated cases were defined as patients who were treated for TB for 1 month or more without considering chemoprophylaxis $[7,20]$. The fourth characteristic considered was program related factor which included the year of enrolment.

\section{Data processing and analysis}

After the data was checked for its consistency and completeness, it was entered in to epi-data version 4.2.0.0 and exported to Stata version 14.1 software for further cleaning and analysis. Summary statistics were carried out to describe demographic, behavioural, clinical, and programmatic data. Incidence rate was calculated by dividing the total number of LTFU to the total person months of observations. Life table was used to estimate cumulative failure at a different point in time. The Nelson-Aalen cumulative hazard curve was employed to estimate overall failure rate. The log-rank test was used to compare survival experience among different exposure groups. The best fitted model was selected based on the highest Likelihood (LL) and lowest information criteria (AIC and BIC), and the Gompertz regression model was fitted. The Cox proportional hazard assumption was tested for each variable and globally by using Schoenfeld residuals. The Nelson-Aalen cumulative hazard verses the Cox-Snell residual graph was used to test the goodness of the model fit. The bivariable model was fitted first and variables with a $p$-value less than or equal to 0.25 were used in the final multivariable model to identify the predictor variables. Finally, the Adjusted Hazard Ratio (AHR) with a 95\% confidence interval (CI) in the multivariable model was used to select independent predictor variables of time to LTFU.

\section{Results \\ Baseline socio-demographic and behavioural characteristics}

A total of 341 patients who started treatment between 2010 and 2017 were eligible for this study of which 9 patients were excluded because five patient charts were lost and four patient outcomes were not evaluated. Therefore, 332 patient records were eligible for analysis; among these, $206(62.05 \%)$ of the patients were male. The median age of the total participants was 30 years (IQR: 23-40), and the median age for lost patients was 29 (IQR: 25-45) years. Three hundred twelve (93.98\%) of the participants were

Table 1 Definitions of treatment outcome for drug-resistant tuberculosis

\begin{tabular}{ll}
\hline Outcome & Definition \\
\hline Cure & $\begin{array}{l}\text { Treatment completed according to national recommendation without evidence of failure and three or more consecutive cultures } \\
\text { taken at least } 30 \text { days apart are negative after the intensive phase. }\end{array}$ \\
Completed & $\begin{array}{l}\text { Treatment completed according to national recommendation without evidence of failure but no record that three or more } \\
\text { consecutive cultures taken at least } 30 \text { days apart are negative after the intensive phase. }\end{array}$ \\
Treatment failure & $\begin{array}{l}\text { Treatment terminated or need for permanent regimen change of at least two anti-TB drugs because of lack of conversion by the end } \\
\text { of the intensive phase, or bacteriological reversion in the continuation phase after conversion to negative after intensive phase, or } \\
\text { evidence of additional acquired resistance to fluoroquinolones or second line injectable drugs, or Adverse drug reactions. } \\
\text { Died } \\
\text { Not evaluated }\end{array} \quad \begin{array}{l}\text { A patient who dies for any reason during the course of TB treatment. } \\
\text { reporting unit. }\end{array}$ \\
\hline
\end{tabular}


from the Amhara Regional State. Nearly 40\% (131) of the participants were married (Table 2).

The total number of DR-TB patients and number of lost patients on treatment gradually increased from 2010 to 2013 and then declined unevenly (Fig. 1).

\section{Baseline clinical status of the participants}

More than 67\% (225) of the cases were ambulatory. Nearly $72 \%$ had a baseline body mass index of less than $18.5 \mathrm{~kg} / \mathrm{m}^{2}$. A quarter of patients $(84 / 330)$ that had test results for HIV were positive. Among those who had recorded results, closely all $(92.17 \%)$ of patients had pulmonary tuberculosis with baseline smear and culture positivity of $84.31 \%(258 / 306)$ and $71.71 \%(180 / 251)$ respectively (Table 3 ).

\section{Lost to follow-up of patients on DR-TB treatment}

Patients were followed for a minimum of 0.33 and a maximum of 27.30 months with a total of 5564.67 personmonths (PM) of observations. The median follow-up time was 20.37 months (IQR: 11.02-21.80). Regarding treatment outcome, 177 (53.31\%) patients were cured, 35 (10.54\%) completed treatment, 36 (10.84\%) died, 36 (10.84\%) LTFU, 40 (12.05\%) on treatment, 2 (0.60\%) transferred out, and $6(1.81 \%)$ had treatment failure. The overall proportion of LTFU was 10.84\% (95\% CI: 7.91, 14.69). The proportions of LTFU among all lost patients were $22,56,67$, and $81 \%$ at the end of the 3rd, 6th, 8 th, and 10th months of follow-up, respectively.

The overall incidence rate of LTFU was 6.47 (95\% CI: $4.67,8.97) / 1000$ person-month (PM) observation. The incidence rate per 1000 person-month of observation was 105.39 [95\% CI: 67.99, 163.36], 34.93 [95\% CI: 18.79, 64.89], 9.65 [95\% CI: 3.62, 25.72] and 0.48 [95\% CI: 0.12, 1.94 ] in the first 6 th, 7 th-12th, 13th-18th, and 19th24th months, respectively. Moreover, the incidence rates per 1000 person-months of observation were 3.20 [95\% CI: 1.44, 7.12], 10.36 [95\% CI: 6.82, 15.74], and 5.11 [95\% CI: $2.55,10.21]$ before the year 2013, from 2013 to 2014, and after 2014 respectively.

The cumulative probability of LTFU was $6.37 \%$ (95\% CI: $4.16,9.70)$ at the 6th month, 9.90\% (95\% CI: 7.02, 13.87) 12th month, $11.45 \%$ (95\% CI: 8.31, 15.68) 18th month, and $12.92 \%$ (95\% CI: 9.37, 17.69) at the 24th month after the start of the follow-up (Fig. 2).

\section{Predictors of lost to follow-up}

A log-rank test $\left(\mathrm{X}^{2}\right)$ of equality of hazard of incidence of LTFU was done for the different categories of explanatory variables. The test result indicated a significant difference in the incidence of lost to follow-up between patients who were homeless and who had home, $p$ value $=0.0046$, and patents who were enrolled before
Table 2 Baseline socio-demographic and behavioural characteristics of DR-TB patients stratified by lost to follow-up, University of Gondar Comprehensive Specialized Hospital, September 2010-December 2017, ( N = 332)

\begin{tabular}{|c|c|c|c|}
\hline \multirow[t]{2}{*}{ 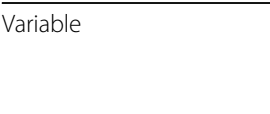 } & \multirow[t]{2}{*}{ Frequency (\%) } & \multicolumn{2}{|c|}{ Lost to follow-up Status } \\
\hline & & $\begin{array}{l}\text { Event }^{\mathrm{a}} \\
(n=36)\end{array}$ & $\begin{array}{l}\text { Censored }^{\mathrm{b}} \\
(n=296)\end{array}$ \\
\hline \multicolumn{4}{|l|}{ Sex } \\
\hline Male & $206(62.05)$ & 20 & 186 \\
\hline Female & $126(37.95)$ & 16 & 110 \\
\hline \multicolumn{4}{|l|}{ Residency } \\
\hline Urban & $169(50.90)$ & 19 & 150 \\
\hline Rural & $163(49.10)$ & 17 & 146 \\
\hline \multicolumn{4}{|l|}{ Region } \\
\hline Amhara & 312 (93.98) & 30 & 282 \\
\hline Tigray & $19(5.72)$ & 6 & 13 \\
\hline Benshangul Gumuz & $1(0.30)$ & 0 & 1 \\
\hline \multicolumn{4}{|l|}{ Marital status } \\
\hline Married & 131 (39.46) & 18 & 113 \\
\hline Single & $104(31.33)$ & 10 & 96 \\
\hline Divorced & $36(10.84)$ & 4 & 32 \\
\hline Widowed & $9(2.71)$ & 2 & 7 \\
\hline Separated & $29(8.74)$ & 1 & 28 \\
\hline$<18$ years & $19(5.72)$ & 2 & 17 \\
\hline Not recorded & $4(1.20)$ & 1 & 3 \\
\hline \multicolumn{4}{|l|}{ Level of education } \\
\hline Not formally educated & $120(36.14)$ & 16 & 104 \\
\hline Primary & $104(31.33)$ & 11 & 93 \\
\hline Secondary & $54(16.27)$ & 6 & 48 \\
\hline Tertiary & $44(13.25)$ & 2 & 42 \\
\hline Not in school age & $5(1.51)$ & 0 & 5 \\
\hline Not recorded & $5(1.51)$ & 1 & 4 \\
\hline \multicolumn{4}{|l|}{ Housing condition } \\
\hline Homeless & $46(13.85)$ & 10 & 36 \\
\hline Having Home & $248(74.70)$ & 26 & 222 \\
\hline Not recorded & $38(11.45)$ & 0 & 38 \\
\hline \multicolumn{4}{|l|}{ Occupation } \\
\hline Not employed & 117 (35.24) & 9 & 108 \\
\hline Employed & 209 (62.95) & 26 & 183 \\
\hline Not recorded & $6(1.81)$ & 1 & 5 \\
\hline \multicolumn{4}{|l|}{ Smoking } \\
\hline No & 277 (83.43) & 32 & 245 \\
\hline Yes & $54(16.27)$ & 3 & 51 \\
\hline Not recorded & $1(0.30)$ & 1 & 0 \\
\hline \multicolumn{4}{|l|}{ Alcohol drink } \\
\hline No & $279(84.04)$ & 30 & 249 \\
\hline Yes & $50(15.06)$ & 5 & 45 \\
\hline Not recorded & $3(0.90)$ & 1 & 2 \\
\hline
\end{tabular}

Event ${ }^{\mathrm{a}}$ in this study was patients lost during follow-up; Censored ${ }^{\mathrm{b}}$ was either cured, completed, death, transfer out, still on treatment 


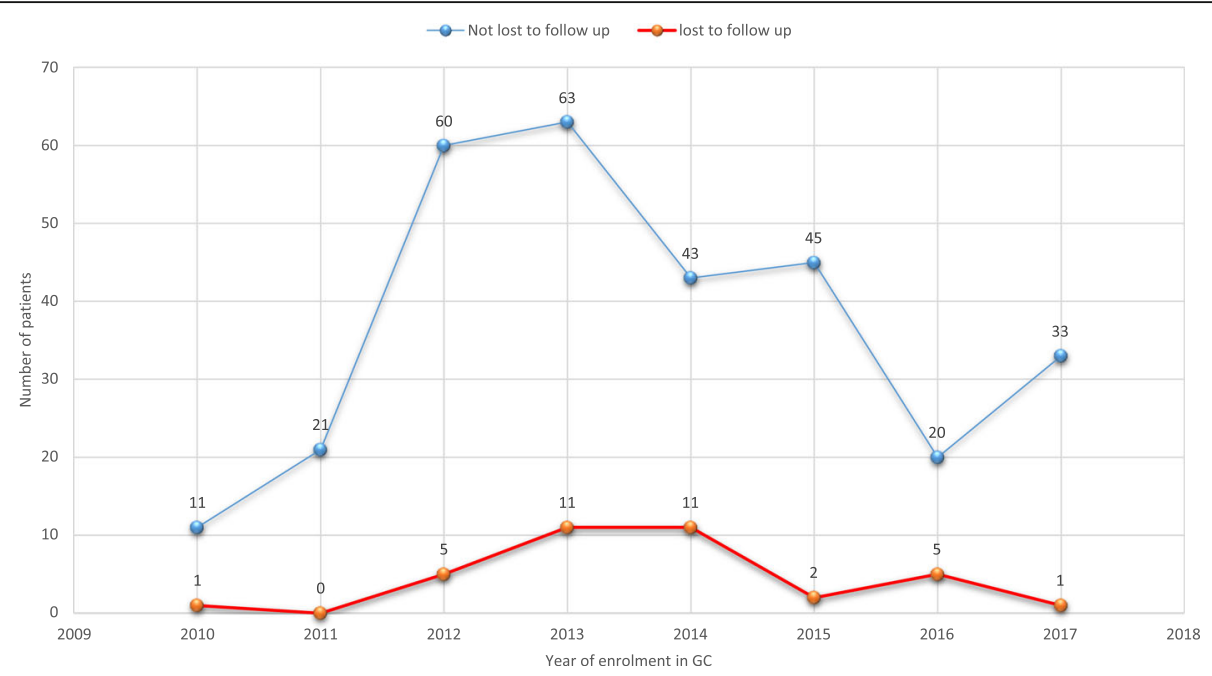

Fig. 1 Trend of the number of DR-TB patients lost and not lost to follow up by year of treatment enrolment at University of Gondar Comprehensive Specialized Hospital, September 2010-December 2017

2013, from 2013 to 2014 and after 2014, $p$-value $=$ 0.0152 at $5 \%$ level of significance (Figs. 3 and 4 ).

The proportional-hazards assumption was assessed based on the Schoenfeld residuals, and it was found that all of the covariates and the full model satisfied the proportional hazard assumption with the global test, $p$ value $=0.8423$.

According to the information criteria (AIC, BIC) and LL comparison techniques, the Gompertz regression model was found to be more efficient than the other semi-parametric and parametric models fitted (Table 4).

In the bivariable Gompertz regression analysis, age, housing condition, treatment start year, cigarette smoking, and occupation were associated with lost to follow-up. However, in the multivariable Gompertz regression analysis, housing condition and treatment start year remained statistically significant predictors of LTFU.

The risk of lost to follow-up among homeless patients was two and half times at higher hazard of LTFU compared to those who had home $(\mathrm{AHR}=2.51,95 \% \mathrm{CI}: 1.15$, 5.45). Patients who started treatment in the year 2013 and 2014 were more than three times at higher hazard of lost to follow-up than those who started treatment before 2013 (AHR = 3.25, 95\% CI: 1.30, 8.13) (Table 5).

Goodness of fit for the selected model was assessed using the Cox-Snell residual test, and the result showed the model provided the best fit for our data (Fig. 5).

\section{Discussion}

This study was designed to assess the incidence rate and to identify the predictors of time to lost to follow- up among DR-TB patients attending their treatment at the University of Gondar Comprehensive Specialized Hospital. The incidence rate of lost to follow-up was found to be 6.47 with 95\% CI: [4.67, 8.97] per $1000 \mathrm{PM}$ of observation, i.e. if we followed 1000 DR-TB patients for a month during their treatment, 6.47 patients were lost from follow-up at the end of the month. The incidence rate per $1000 \mathrm{PM}$ of observation in every 6 month interval (the first $6,7-12,13-18$, and 19-24) was $105.39,34.93,9.65$ and 0.48 respectively, i.e. incidence rate of LTFU was higher in the first 6 months compared to subsequent follow-up months. This finding showed that the incidence rate of LTFU was a challenge to end the epidemic of TB targeted by SDGs and the End TB strategy by 2030 and 2035, respectively [13, 21 in the study area and similar settings. From the total lost to follow-up patients, 22.2\% (8/36) and 55.6\% $(20 / 36)$ were lost in the first three and 6 months of treatment, respectively. This finding was low compared to a finding from India which showed more than half were lost in the third [22] and $86.9 \%$ in the sixth [17] months of treatment. On the other, hand the finding was higher compared to that of a study in South Africa which showed $8.19 \%$ of the patients were lost in the third month of treatment [23]. These differences might be due to differences in the mode of treatment delivery in that the study from India was based on the ambulatory model of care (without initial hospitalization), and the possible reason for the high lost in the South African study might be the use of the community level study design. In general, the likely explanation for patients to be lost in the early period of treatment might be due to adverse drug effects commonly associated 
Table 3 Baseline clinical characteristics of DR-TB patients stratified by lost to follow-up status, University of Gondar Comprehensive Specialized Hospital, September 2010-December 2017, ( $N=332)$

\begin{tabular}{lll}
\hline Variable & Frequency (\%) & Lost to follow-up \\
& status & \\
\cline { 2 - 3 } & $\begin{array}{lll}\text { Event }^{\mathrm{a}} & \text { Censored }^{\mathrm{b}} \\
& (n=36) & (n=296)\end{array}$ \\
\hline
\end{tabular}

$\mathrm{BMI}$

$$
<18.5
$$$$
>=18.50
$$

$221(71.99)$

25

186

Functional status

$86(18.01)$

$$
8
$$

78

\section{Working}

Ambulatory

Bedridden

Registration Group

New
Relapse
Return after lost to follow-up
Failure

outcome not assigned

No of previous TB treatment

$<2$

Corpulmonale

Bronchiectasis

Co-morbidity

$$
\text { Yes }^{\text {d }}
$$

HIV co-infection

No
Yes
Unknown

Base line sputum smear result

$$
\begin{aligned}
& \text { Negative } \\
& \text { Positive }
\end{aligned}
$$

Not recorded

Baseline Culture result

Positive

Negative
78 (23.49)

$225(67.77)$

7

29 (8.73)

41 (12.35)

45 (13.55)

$2(0.60)$

$241(72.60)$

$3(0.90)$

$112(33.74)$

$218(65.66)$

$2(0.60)$

$306(92.17)$

$26(7.83)$

$275(82.83)$

$39(11.75)$

5 (1.51)

9 (2.71)

\section{$4(1.20)$}

$277(83.43)$

$55(16.57)$

246 (74.10)

$84(25.30)$

$2(0.60)$

71

196

29

37

40

1 215

3

\begin{tabular}{|c|c|c|c|}
\hline \multirow[t]{2}{*}{ Variable } & \multirow[t]{2}{*}{ Frequency (\%) } & \multicolumn{2}{|c|}{$\begin{array}{l}\text { Lost to follow-up } \\
\text { status }\end{array}$} \\
\hline & & $\begin{array}{l}\text { Event }^{a} \\
(n=36)\end{array}$ & $\begin{array}{l}\text { Censored }^{\mathrm{b}} \\
(n=296)\end{array}$ \\
\hline Not recorded & $81(24.40)$ & 9 & 72 \\
\hline \multicolumn{4}{|c|}{ Culture converted among baseline Culture result positive } \\
\hline Yes & 159 (88.33) & 9 & 150 \\
\hline No & $21(11.67)$ & 6 & 15 \\
\hline \multicolumn{4}{|l|}{ DR-TB type } \\
\hline RR-TB & $147(44.28)$ & 17 & 130 \\
\hline MDR-TB & $168(50.60)$ & 18 & 150 \\
\hline Clinically diagnosed & $17(5.12)$ & 1 & 16 \\
\hline
\end{tabular}

Table 3 Baseline clinical characteristics of DR-TB patients stratified by lost to follow-up status, University of Gondar Comprehensive Specialized Hospital, September 2010-December 2017, ( $N=332)$ (Continued)

with injectable drugs mostly taken during the first phase of treatment [14, 16, 24]. This could make exhaust and urge patients to interrupt their treatments due to repeated treatments with first line anti-TB drugs which in turn leads patients to be lost from follow-up $[14,16,24]$. Among lost patients, $86 \%$ were pulmonary TB cases, and out of those who had data on culture result at the time of lost to follow-up, $40 \%$ were not culture converted. This finding was similar to a finding in a Georgian study (40\%) [24]. Patients with positive culture result are very infectious and puts communities at risk for developing primary DR-TB. The above findings illustrate the challenges to achieving completion of the recommended regimens for DR-TB and the TB prevention strategy at that movement $[14,24]$. Evidence from different high DR-TB burden countries noted that low default rates or good DR-TB treatment success rates were related to the implementation of communitybased treatment practices which were not yet implemented in Ethiopia. A community-based treatment practice is an effective strategy for increasing access to treatment which is patient centered and more acceptable to patients in that it has a great contribution to the retention of patients in the care $[23,25,26]$.

In this study, we tried to assess factors which predicted the time to LTFU and noted that homelessness and year of treatment enrolment remained independent predictors for lost LTFU.

The hazard of lost to follow-up among homeless patients was two and half times higher compared to those who had home. Similar association were reported by studies in rural South Africa [12], Limu Peru [27], and 


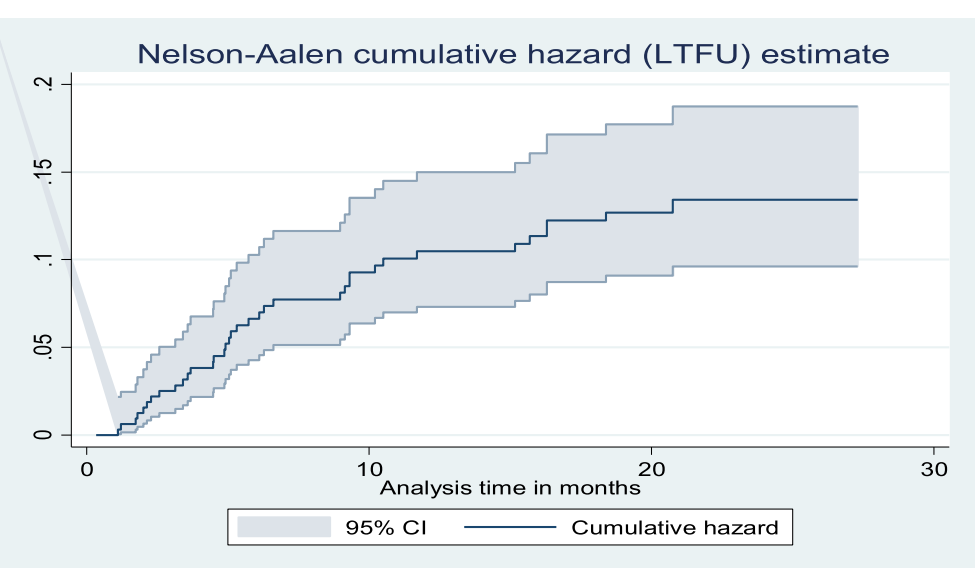

Fig. 2 Nelson-Aalen cumulative hazard estimation of LTFU of patients under DR-TB Treatment at University of Gondar Comprehensive Specialized Hospital, September 2010-December 2017

one meta-analysis [28]. Despite the provision of individualized socioeconomic support to all patients by the national Programmatic Management of Drug-resistant Tuberculosis (PMDT) program, LTFU among homeless patients was high. This might be explained by the fact that homelessness is extremely linked to poverty that triggers the risk for LTFU [29]. Another reason could be the difficulty of tracing such patients when they interrupt treatment. Moreover, it can also be explained in that majority $(67.47 \%)$ of our patients were in the age of thirties who might be responsible to support their families that could trigger to lost from follow-up. The relationship between financial constraints, adequate nutrition, and the fear of losing jobs were challenges to patients who need to complete treatments as evidenced by different studies [14, 29]. Therefore, the disparity between working hours and treatment center schedules as well as inability to work due to treatment side effects might be the possible reasons for the higher hazard of LTFU among homeless patients.

Patients who started treatment in the years 2013 to 2014 were more than three times at higher hazard of lost to follow-up compared to those who started treatment before 2013. This finding was supported by those of studies done in Georgia [24] and Limu, Peru [27]. This can be justified by the fact that in 2013 to 2014 years of treatment enrolments the rise in the number of LTFU was thought to be secondary in light of the decreasing financial support and the need to spreading the money over the increasing number of patients. In addition, it was also believed to be secondary to the decreasing ability to provide individualised patient-

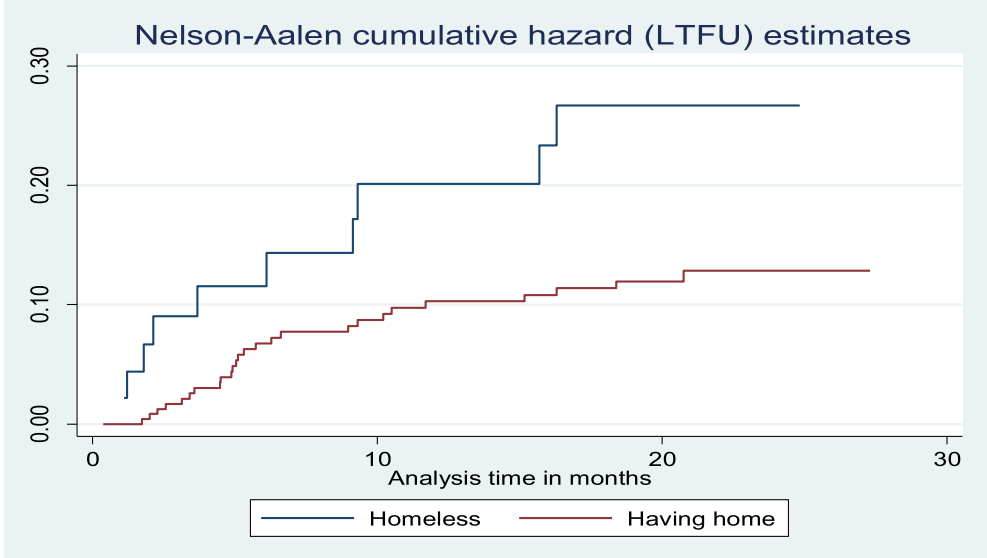

Fig. 3 Nelson-Aalen cumulative hazard curve of lost to follow up by housing status on time to LTFU among DR-TB patients at University of Gondar Comprehensive Specialized Hospital, September 2010-December 2017 


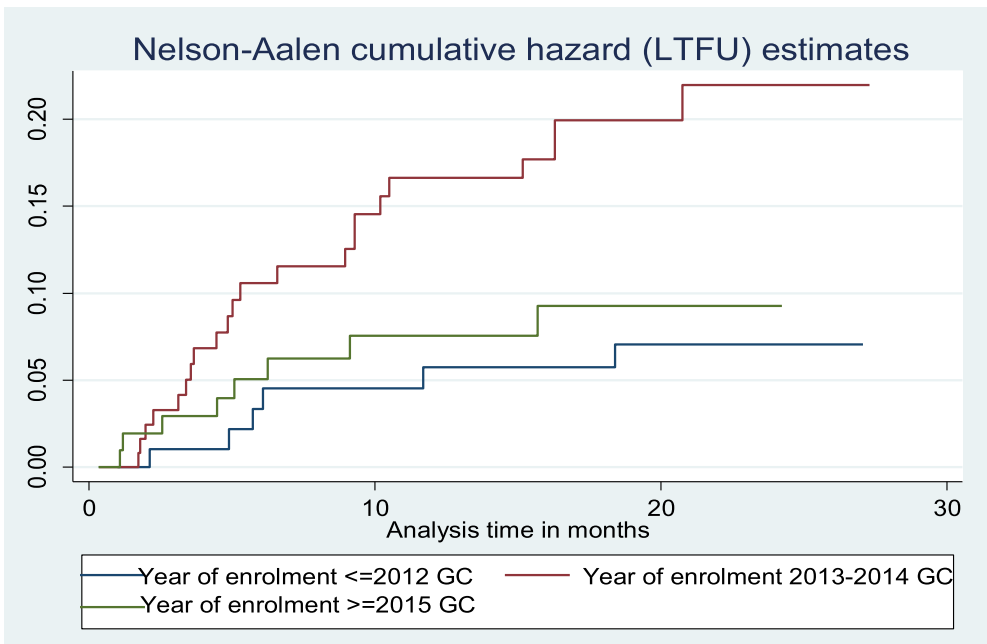

Fig. 4 Nelson-Aalen cumulative hazard curve of lost to follow up by year of treatment start on time to LTFU among DR-TB patients at University of Gondar Comprehensive Specialized Hospital, September 2010-December 2017

centred care as the total DR-TB cohort size increased. Although previous studies showed that decentralized care appeared to be more likely to decreased lost to follow-up [26, 30], during the year 2013 to 2014 the Ethiopian National Tuberculosis Control Program (NTP) implemented a new PMDT guideline that promoted the decentralization of DR-TB services, and patients were linked to the newly established treatment initiating centers in the region. During linkage to the new sites, poor patient preparation and unequally equipped new sites might have increased the number of lost patients. The medical staff at that time witnessed that during the decentralization patients were linked from highly equipped, multi-disciplinary, more experienced, and more socioeconomically supporting site, to less experienced, new sites which dissatisfied patients by the services. Furthermore, the clinical team also had chances of visiting the new DR-TB TICs and communicating with some of the lost patients and confirmed that the newly developed treatment sites were primary

Table 4 Summary of model comparison between Cox proportional hazard model and parametric survival distribution models using likelihood (LL) and information criteria (AIC and BIC)

\begin{tabular}{|c|c|c|c|c|c|}
\hline \multirow{2}{*}{$\begin{array}{l}\text { Comparison } \\
\text { Method }\end{array}$} & \multicolumn{5}{|l|}{ Models } \\
\hline & $\mathrm{COXPH}$ & Weibull & Exponential & Gompertz $^{a}$ & $\begin{array}{l}\text { Log } \\
\text { logistic }\end{array}$ \\
\hline$\overline{\mathrm{LL}}$ & $\begin{array}{l}- \\
183.4165\end{array}$ & $\begin{array}{l}- \\
139.9643\end{array}$ & -140.8364 & -136.894 & $\begin{array}{l}- \\
139.3641\end{array}$ \\
\hline $\mathrm{AlC}$ & 378.833 & 295.9287 & 295.6728 & 289.7879 & 294.7281 \\
\hline $\mathrm{BIC}$ & 400.8316 & 325.2601 & 321.3378 & 319.1193 & 324.0596 \\
\hline
\end{tabular}

${ }^{\mathrm{a}}$ Highest $\mathrm{LL}$ and lowest AIC and BIC hospitals with lots of shortcomings compared to the University of Gondar hospital. The University of Gondar Hospital DR-TB treatment site team were composed of multi-discipline teams (physicians, nurses, social workers, psychiatrist, expert patients, and other supporting non-governmental organizations like Global Health Committee).

Lost to follow-up in DR TB patients is an important routine indicator of the achievement of better treatment outcomes. Preventing lost from DR-TB treatment can improve patient intended treatment outcome, future health status, and prevent further drug resistant strains of tuberculosis. In addition, it is vital in TB infection control strategy since patients with DR-TB and positive sputum result are infectious and may transmit the disease to the people at risk in the general community. Despite the greet efforts we made to estimate the incidence and identify the predictors of LTFU among DR-TB patients our study has some limitations, since we used secondary data, some important sociodemographic, bacteriological, and economic factors which might have effects on LTFU could have been missed. Besides, the small number of patients still on treatment at the end of study who have the chance of LTFU might have under estimated the incidence.

\section{Conclusion}

This study found that lost to follow-up among DR-TB registered patients for treatment was high in the first 6 months compared to later follow-up months. Housing status and year of treatment enrolment were independent predictors of time to LTFU. This result could be 
Table 5 Bivariable and multivariable Gompertz regression analysis for predictors of time to lost to follow-up among DR-TB patients, University of Gondar Comprehensive Specialized Hospital, September 2010-December 2017, ( $=332)$

\begin{tabular}{|c|c|c|c|c|}
\hline Variable & Event $^{a}$ & Censored $^{\mathrm{b}}$ & CHR $(95 \% \mathrm{Cl})$ & AHR $(95 \% \mathrm{Cl})$ \\
\hline Median Age (IQR) & $29(25,45)$ & $30(22,40)$ & $1.02(1.00,1.05)$ & $1.03(0.99,1.05$ \\
\hline \multicolumn{5}{|l|}{ Sex } \\
\hline Male & 20 & 186 & 1 & \\
\hline Female & 16 & 110 & $1.34(0.69,2.58)$ & \\
\hline \multicolumn{5}{|c|}{ Body mass index $\left(\mathrm{kg} / \mathrm{m}^{2}\right)$} \\
\hline$<18.5$ & 25 & 196 & $1.22(0.552 .71)$ & \\
\hline$>=18.50$ & 8 & 78 & 1 & \\
\hline \multicolumn{5}{|l|}{ Housing Condition ${ }^{c}$} \\
\hline Homeless & 10 & 36 & $2.20(1.06,4.57)$ & $2.51(1.15,5.45$ \\
\hline Having Home & 26 & 222 & 1 & 1 \\
\hline \multicolumn{5}{|l|}{ Alcohol drink } \\
\hline No & 30 & 249 & 1 & \\
\hline Yes & 5 & 45 & $0.91(0.35,2.35)$ & \\
\hline \multicolumn{5}{|l|}{ Co-morbidity } \\
\hline No & 30 & 247 & 1 & \\
\hline Yes & 6 & 49 & $1.12(0.46,2.68)$ & \\
\hline \multicolumn{5}{|c|}{ Year of treatment initiation ${ }^{c}$} \\
\hline$<=2012$ & 6 & 92 & 1 & 1 \\
\hline 2013-2014 & 22 & 106 & $3.08(1.25,7.59)$ & $3.25(1.30,8.13$ \\
\hline$>=2015$ & 8 & 98 & $1.42(0.49,4.11)$ & $2.07(0.71,5.99$ \\
\hline
\end{tabular}

Base line sputum smear result

$\begin{array}{ll}\text { Negative } & 6 \\ \text { Positive } & 27\end{array}$

42

231

Residency

Urban

Rural

Cigarette smoking

No

Yes

HIV co-infection

$\begin{array}{ll}\text { No } & 27 \\ \text { Yes } & 9\end{array}$

Number of previous TB treatment

$\begin{array}{ll}<2 & 11 \\ >=2 & 25\end{array}$

Occupation

Employed

146

$0.97(0.51,1.86)$

$0.77(0.32,1.87)$
101

193

108
1

$1.05(0.49,2.24)$
26

Unemployed

9

$\mathrm{LR} \mathrm{Chi}^{2}(6)=17.14$ probability $>\mathrm{chi}^{2}=0.0088$

CHR Crude Hazard ratio, AHR Adjusted Hazard Ratio, Cl Confidence Interval

Event ${ }^{\mathrm{a}}$ in this study was patients lost during follow-up; Censored ${ }^{\mathrm{b}}$ was either cured, completed, death, transfer out, still on treatment

The shape parameter gamma was found to be -0.0811245 ( $95 \% \mathrm{Cl}:-0.1407007,-0.0215482)$ which is negative. This indicates that the hazard of lost to follow-up decreases exponentially with time

'Significantly associated variables in both bivariable and multivariable model 


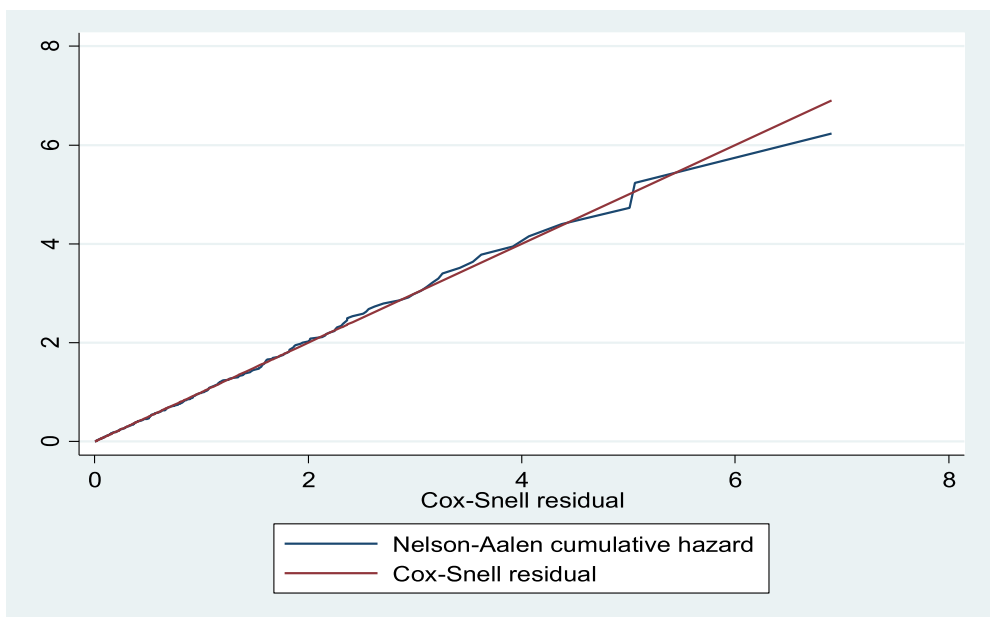

Fig. 5 Nelson-Aalen cumulative hazard against Cox-Snell residual plot for Gompertz model for LTFU among DR-TB patients at University of Gondar Comprehensive Specialized Hospital, September 2010 - December 2017

generalized to patients who are using DR-TB treatment in a similar setting in Ethiopia. There is a need for screening and prioritising risk factors and developing programmatic strategies to provide comprehensive socioeconomic support (incentives and/or enablers) to socioeconomically underprivileged patients in order to ensure treatment success.

\section{Abbreviations}

DR-TB: Drug Resistance Tuberculosis; LTFU: Lost To Follow-up; MDR-TB: MultiDrug Resistant Tuberculosis; PM: Person Months; PMDT: Programmatic Management of Drug-resistant Tuberculosis; RR-TB: Rifampicin Resistance Tuberculosis; SDGs: Sustainable Development Goals; TB: Tuberculosis; TIC: Treatment Initiation Center; WHO: World Health Organization

\section{Acknowledgements}

We are grateful to the data collectors for their precious time spent during data collection. We also acknowledge the University Gondar hospital for permitting us to conduct the study.

\section{Authors' contributions}

GMK incepted and designed study, participated in the data collection supervision, performed analysis, and interpretation of data and drafted the paper and revised the manuscript. AST and HFW assisted the designing of the study, developed the method section of the paper, and revised drafts of the paper, and worked on the manuscript. MWM and AGM assisted in the analysis and interpretation of the findings, drafting the manuscript. All authors read and approved the final manuscript.

\section{Funding}

None.

\section{Availability of data and materials}

The datasets used during the current study are available from the corresponding authors upon reasonable request and with permission of University of Gondar Comprehensive Specialized Hospital.

\section{Ethics approval and consent to participate}

Ethical clearance was obtained from Institutional Review Board of College of Medicine and Health Sciences, University of Gondar. Permission letter was obtained from Amhara Public Health Institute, the Hospital administration, and the TB ward focal person. Personal identifiers were not included during data collection.
Consent for publication

Not applicable.

\section{Competing interests}

The authors declare that they have no competing interests.

Received: 18 December 2018 Accepted: 9 September 2019

Published online: 18 September 2019

\section{References}

1. World Health Organization. Global strategy and targets for tuberculosis prevention, care and control after 2015. Geneva: WHO; 2015. Contract No: EB134/12. 2017

2. World Health Organization. Global tuberculosis report. 2016.

3. World Health Organization. Global tuberculosis report 2018.

4. World Health Organization. Global tuberculosis report; 2015. p. 9241565055.

5. World Health Organization. Global tuberculosis report 2017.

6. Lukoye D, Ssengooba W, Musisi K, Kasule GW, Cobelens FG, Joloba M, et al. Variation and risk factors of drug resistant tuberculosis in sub-Saharan Africa: a systematic review and meta-analysis. BMC Public Health. 2015;15(1):291.

7. FDRE MoH. gaideline on programmatic management of drug resistant tuberculosis in ethiopia. 2017.

8. Eshetu L, Beniam F, Abebaw K, Muluwork G, Zelalem Y, Ribka F, et al. Second round anti-tuberculosis drug resistance surveillance in Ethiopia,. 2014.

9. Eshetie S, Gizachew M, Dagnew M, Kumera G, Woldie H, Ambaw F, et al. Multidrug resistant tuberculosis in Ethiopian settings and its association with previous history of anti-tuberculosis treatment: a systematic review and meta-analysis. BMC Infect Dis. 2017;17(1):219.

10. Tessema B, Beer J, Emmrich F, Sack U, Rodloff A. First-and second-line antituberculosis drug resistance in Northwest Ethiopia. Int J Tuberc Lung Dis. 2012;16(6):805-11.

11. World Health Organization. Global tuberculosis report 2014.

12. Kendall EA, Theron D, Franke MF, Van Helden P, Victor TC, Murray MB, et al. Alcohol, hospital discharge, and socioeconomic risk factors for default from multidrug resistant tuberculosis treatment in rural South Africa: a retrospective cohort study. PLoS One. 2013;8(12):e83480.

13. Uplekar M, Weil D, Lonnroth $\mathrm{K}$, Jaramillo $\mathrm{E}$, Lienhardt C, Dias HM, et al. WHO's new end TB strategy. Lancet. 2015;385(9979):1799-801.

14. Deshmukh RD, Dhande D, Sachdeva KS, Sreenivas A, Kumar A, Satyanarayana S, et al. Patient and provider reported reasons for lost to follow up in MDRTB treatment: a qualitative study from a drug resistant TB Centre in India. PLoS One. 2015;10(8):e0135802.

15. Tupasi TE, Garfin AMCG, Kurbatova EV, Mangan JM, Orillaza-Chi R, Naval LC, et al. Factors associated with loss to follow-up during treatment for multidrug-resistant tuberculosis, the Philippines, 2012-2014. Emerg Infect Dis. 2016;22(3):491. 
16. Wohlleben J, Makhmudova M, Saidova F, Azamova S, Mergenthaler C, Verver S. Risk factors associated with loss to follow-up from tuberculosis treatment in Tajikistan: a case-control study. BMC Infect Dis. 2017;17(1):543.

17. Shringarpure KS, Isaakidis P, Sagili KD, Baxi R. Loss-to-follow-up on multidrug resistant tuberculosis treatment in Gujarat, India: the when and who of it. PLoS One. 2015;10(7):e0132543.

18. Alene KA, Viney K, McBryde ES, Tsegaye AT, Clements AC. Treatment outcomes in patients with multidrug-resistant tuberculosis in north-West Ethiopia. Tropical Med Int Health. 2017;22(3):351-62.

19. Meressa D, Hurtado RM, Andrews JR, Diro E, Abato K, Daniel T, et al. Achieving high treatment success for multidrug-resistant TB in Africa: initiation and scale-up of MDR TB care in Ethiopia-an observational cohort study. Thorax. 2015. https://doi.org/10.1136/thoraxjnl-2015-207374.

20. Falzon D, Schünemann HJ, Harausz E, González-Angulo L, Lienhardt C, Jaramillo $\mathrm{E}$, et al. World Health Organization treatment guidelines for drugresistant tuberculosis, 2016 update. Eur Respir J. 2017;49(3):1602308.

21. World Health Organization. World health statistics 2016: monitoring health for the SDGs sustainable development goals. Geneva: World Health Organization; 2016.

22. Suryawanshi S, Shewade H, Nagaraja S, Nair S, Parmar M. Unfavourable outcomes among patients with MDR-TB on the standard 24-month regimen in Maharashtra, India. Public Health Action. 2017;7(2):116-22.

23. Moyo S, Cox HS, Hughes J, Daniels J, Synman L, De Azevedo V, et al. Loss from treatment for drug resistant tuberculosis: risk factors and patient outcomes in a community-based program in Khayelitsha, South Africa. PLoS One. 2015;10(3):e0118919.

24. Kuchukhidze G, Kumar A, de Colombani P, Khogali M, Nanava U, Blumberg H, et al. Risk factors associated with loss to follow-up among multidrug-resistant tuberculosis patients in Georgia. Public Health Action. 2014;4(2):S41-S6.

25. Cox H, Hughes J, Daniels J, Azevedo V, McDermid C, Poolman M, et al. Community-based treatment of drug-resistant tuberculosis in Khayelitsha, South Africa. Int J Tuberc Lung Dis. 2014;18(4):441-8.

26. Toczek A, Cox H, Du Cros P, Cooke G, Ford N. Strategies for reducing treatment default in drug-resistant tuberculosis: systematic review and meta-analysis. Int J Tuberc Lung Dis. 2013;17(3):299-307.

27. Franke MF, Appleton SC, Bayona J, Arteaga F, Palacios E, Llaro K, et al. Risk factors and mortality associated with default from multidrug-resistant tuberculosis treatment. Clin Infect Dis. 2008;46(12):1844-51.

28. Kurbatova EV, Taylor A, Gammino VM, Bayona J, Becerra M, Danilovitz M, et al. Predictors of poor outcomes among patients treated for multidrug-resistant tuberculosis at DOTS-plus projects. Tuberculosis. 2012;92(5):397-403.

29. Tupasi T, Garfin A, Mangan J, Orillaza-Chi R, Naval L, Balane G, et al. Multidrug-resistant tuberculosis patients' views of interventions to reduce treatment loss to follow-up. Int J Tuberc Lung Dis. 2017;21(1):23-31.

30. Ho J, Byrne AL, Linh NN, Jaramillo E, Fox GJ. Decentralized care for multidrug-resistant tuberculosis: a systematic review and meta-analysis. Bull World Health Organ. 2017;95(8):584

\section{Publisher's Note}

Springer Nature remains neutral with regard to jurisdictional claims in published maps and institutional affiliations.

Ready to submit your research? Choose BMC and benefit from:
- fast, convenient online submission
- thorough peer review by experienced researchers in your field
- rapid publication on acceptance
- support for research data, including large and complex data types
- gold Open Access which fosters wider collaboration and increased citations
- maximum visibility for your research: over 100M website views per year
At BMC, research is always in progress.
Learn more biomedcentral.com/submissions

